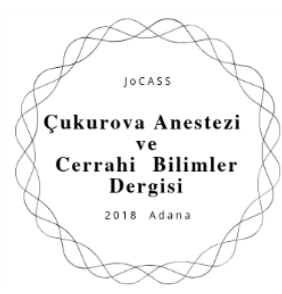

\title{
PERIOPERATIF KARDIYAK ARREST VE MORTALITE
}

\section{PERIOPERATIVE CARDIAC ARREST AND MORTALITY}

\author{
Özge TURGAY YILDIRIM1', Ayşegül TURGAY², ÖZlem AKSOY3, Demet LAFLI TUNAY4 \\ 'Eskişehir Şehir Hastanesi Kardiyoloji Kliniği, Eskişehir \\ ${ }^{2}$ Sinop Atatürk Devlet Hastanesi, Anestezi ve Reanimasyon Kliniği, Sinop \\ Sağlık Bilimleri Üniversitesi, Ankara Çocuk Sağlığı ve Hastalıkları Hematoloji Onkoloji Eğitim ve Araştırma Hastanesi Çocuk Nefroloji Kliniği, \\ Ankara \\ Çukurova Üniversitesi Tıp Fakültesi Anestezi ve Reanimasyon Anabilim Dalı, Adana
}

Sorumlu Yazar/Corresponding Author: Özge Turgay Yıldırım E-mail:özgeturgay@gmail.com

Gelis Tarihi/Received: 14.04.2019 Kabul Tarihi-Accepted: 20.04.2019 Available Online Date/Cevrimiçi Yavın Tarihi: 30.04.2019

Cite this article as: Turgay Yıldırım Ö, Turgay A, Aksoy Ö, Laflı Tunay D, Perioperatif kardiyak arrest ve mortalite. Çukurova Anestezi ve Cerrahi Bilimler Dergisi. 2019:2(1);5-10.

Doi: 10.1XXXXX/JoCASS2019

\section{Özet \\ Perioperatif kardiyak arrest ve mortalite anestezi, cerrahi ve tüm invaziv prosedürlerin en önemli komplikasyonudur. Perioperatif mortalite insidansının yıllar içinde değişimi izlendiğinde düşüş seyri gözlenmektedir. Bu düşüş gelişmişülkelerde belirginken gelişmekte olan ülkelerde istatistiksel anlamlı bir düşüş saptanmamıştır. Perioperatif mortalite açısından en riskli yaş grubu yenidoğan, bir yaş altı çocuklar ve geriatrik hastalardır. Anesteziye bağlı kardiyak arrest ise çoğunlukla hava yolu problemleri ve anestezik ilaçlara sekonder nedenlerle olmaktadır. Bu derleme ile amaçlanan perioperatif ve anesteziye bağıı kardiyak arrest ve mortalitenin yıllar içinde değişimini irdelemek ve risk faktörlerinin göz önüne koymaktır.}

Anahtar Kelimeler: Anestezi, kardiyak arrest, mortalite

\section{Abstract}

Perioperative cardiac arrest and mortality are the most important complications of anesthesia, surgery and all invasive procedures. A decrease in the incidence of perioperative mortality is observed in the course of years. This decline was evident in developed countries but not statistically significant in developing countries. The most risky age group in terms of perioperative mortality was the newborn, children under one year of age and geriatric patients. Anesthesia-related cardiac arrest is mostly due to airway problems and secondary reasons for anesthetic drugs. The aim of this review is to investigate the change pattern of perioperative and anesthesia induced cardiac arrests and mortality over the years and to evaluate the risk factors.

Keywords: Anesthesia, cardiac arrest, mortality

\section{Giriş}

Kardiyak arrest anestezi, cerrahi ve girişimsel prosedürlerin en önemli ve yıkıcı risklerindendir. Perioperatif mortalite cerrahi öncesi, sırası ve sonrasında nadir görülse de üzerinde durulması ve nedenlerinin araştırılması gereken bir konudur ${ }^{1}$. Anesteziye bağlı mortalite ilk kez 1954 yılında araştırılmış ve bu tarihten itibaren ilgili konuda çok sayıda 
araştırma ve yayın yapılmıştır ${ }^{2 \cdot 15}$. Son 50 yılda yapılan çalışmalarda hastaya ait risk faktörleri ve kompleks komorbiditelere rağmen anesteziye bağlı ve genel perioperatif mortalitenin düşmekte olduğu görülmektedir. Intraoperatif kardiyak arrest ile tanımlanan hastaların \%92-96 oranında büyük çoğunluğunu ASA III ve üstü hastalar oluştrmaktadı̊ ${ }^{4,16}$. Bunun yanı sıra gelişmekte olan ülkelerle gelişmiş ülkeler karşılaşırıldığında gelişmekte olan ülkelerde perioperatif mortalite daha yüksek saptanmıştırti, ${ }^{17,18}$.

Anesteziye bağlı kardiyak arrest ve mortalite insidansında düşüşs seyri izlenmektedir. Bunun nedenleri arasinda monitorizasyon tekniklerinin gelişmesi, kılavuz önerilerinin benimsenmesinin yaygınlaşması ve diğer sistematik yaklaşımlar sayılabiliır ${ }^{19,20}$. Anesteziye bağ lı ölümlerle ilgili araştırmalar devam etse de sonuçlarda çelişkili bulgular saptanabilmektedir. Bunun nedeni hastaların risk belirlemesinde yapılan hatalar, operasyon metod ve yaklaşımlarındaki farklılıklara ikincil olabilmektedir ${ }^{3}$. Bu derleme ile perioperatif ve anesteziye bağlı kardiyak arrestler ve mortaliteye yönelik bilgilendirme yapılması amaçlanmıştır.

\section{Anesteziye Bağlı Kardiyak Arrestlerin Ylllara Göre Değişimi}

Anesteziye bağlı kardiyak arrest insidansı tam olarak bilinmemekte ve araştırılmaya devam etmekte olan bir konudur. 2002'de yayınanan bir derleme ASA fiziksel statüsü 1-5 arası hastalarda perioperatif mortalitenin 500 hastada 1 iken, anesteziye bağlı mortalite hızının 13000 hastada 1 olduğunu belirtmiştir ${ }^{3}$. 2003 verilerine göre ise Amerika'da tersiyer bir merkezde perioperatif kardiyak arrest sıklığı 10000 hastada 4,3 iken anesteziye bağlı kardiyak arrest sıklığ 10000 'de 0,5 olarak tespit edilmiştir" ${ }^{11}$ Kurumlar arasında standardizasyon olmadığından veri toplama ve analiz farklı olabilmektedir. Ve kurumlar arası sonuçlarda farklılık olmasının beklenen bir durum olduğu akıldan çıkarılmamalıdır ${ }^{21} .2005$ ve 2007 tarihlerinde 362767 hastanın incelendiği bir çalışmada ise intraoperatif kardiyak arrest sıklı̆g cerrahilerde 10000 hastada 7 olarak saptanmıştır ${ }^{12} .2008$ yayınlanan bir derleme ise perioperatif mortalitenin 13900 hastada 1'e düştüğünü göstermiştir. Bu düşüşteki sebep anestezi öncesi ve sonrası bakımdaki geişme ve iyileşmelere bağlanmıştır²2 2009 yılında Brazilya'da yayınlanan bir meta-analizde perioperatif mortalitenin 10000 hastada 19 ile 51 arasında değiştiği saptanmıştır. Mortalite açısından yenidoğan, 1 yaş altı çocuklar, yaşlı hastalarda risk daha fazla tespit edilmiştir. Yapılan cerrahide intraoperatif ölümler en sık kardiyak cerrahiler sırasında olmakta, bunu torasik, vasküler, gastroenterolojik, pediatrik ve ortopedik cerrahiler takip etmektedir. Anestezik uygulamaya bağlı kardiyak etkiler, kanama ve anemi sonrası yetersiz kan desteği yapılması ve hava yolu yönetim hataları anesteziye bağlı ölümleri başta gelen sebepleri olarak belirlenmiştir ${ }^{23} .2012$ yllında Bainbridge ve arkadaşları tarafından yıllar içinde gelişmiş ve gelişmekte olan ülkelerdeki perioperatif ve 
anesteziye bağlı ölümleri inceleyen bir metaanaliz yayınlanmıştır. Bu çalışmaya göre 1970 yılından önce anestezi sonrası ölümler milyonda 357 iken, 1970-80 arasinda milyonda 52'ye ve 1990-2000 döneminde milyonda 34'e gerilemiştir: bu düşüş istatistiksel olarak anlamlı düzeydedir. $\mathrm{Bu}$ veriler gelişmiş ve gelişmekte olan ülkelere göre incelendiğinde bu iyileşmenin sadece gelişmiş ülkelerde olduğu görülmüştür. Gelişmekte olan ülkelerde hem peroperatif mortalite, hem de anesteziye bağlı mortalitede anlamlı azalma olmadığı saptanmıştır ${ }^{24}$. 2014'te Ellis ve arkadaşlarının çalışmasında anestezi ile kardiyak arrestler 10000 hastada 0,6 olarak tespit edilmiştir. Anesteziye atfedilebilen kardiyak arrestlerin \%64'ü hava yolu kaynaklı komplikasyonlara bağlıdır ve mortalite \%29 saptanmıştır $^{25}$ Sobreira-Fernandes ve arkadaşlarının 122289 hasta verisi üzerinden 2018 yılında yaptıkları araştırmada ise perioperatif kardiyak arrest insidansı 10000 anestezide 5,7 saptanmış olup ilişkili mortalite 10000'de 2,9 olarak saptanmıştır. Bu çalışmada bağımsız risk faktörleri ASA skorunun 3 ve üzerinde olması, kardiyak hastalık öyküsü olması ve vazopressör kullanımı olarak saptanmıştır. Azalmış sağkalım nedenleri de yüksek ASA skoru, acil ve kompleks cerrahiler, vazopressör kullanımı, arrest öncesi hipotansiyon ve kanamaya sekonder kardiyak arrestler olarak belirlenmiştir. Aynı çalışmada anesteziye bağlı kardiyak arrest insidansı ise 10000 anestezide 0,74 ve anesteziye sekonder mortalite 10000 anestezide 0,08 olarak saptanmıştır. Anesteziye bağlı perioperatif kardiyak arrestlerin başlıca nedenleri ilaca bağlı ve havayolu/ventilasyona bağlı komplikasyonlar olarak saptanmıştır. Bağımsız risk faktörleri ise yüksek ASA skoru ve kardiyak hastalık öyküsü olarak belirtilmiştir ${ }^{26}$.

\section{Pediatrik Populasyonda Perioperatif Kardiyak} Arrest ve Ölümlerin İncelenmesi

Yetişkin popülasyonla karşılaştıılddı̆̆ında çocuklarda perioperatif mortalite hızı daha yüksektir ${ }^{2,7,16,27}$. Pediatrik hastalar kendi içinde incelendiğinde ise yenidoğan ve bebeklerde perioperatif mortalitenin daha yüksek olduğu izlenmektedir ${ }^{7,27-31}$. Gelişmiş ülkeler incelendiğinde anesteziye bağlı ölümlerin azalma seyrinde olduğu izlenmiştir. Pediatrik populasyonda anesteziye bağlı ölüm oraları 1961-2000 arasında 10000 anestezide 0,2-2,9 oranında seyretmekte iken 2000-20011 arasinda 10000 anestezide 0,0-0,69 oranına çekildiği izlenmektedir ${ }^{4,29,31-33}$. Gelişmekte olan ülkelerde ise 2001-2011 arasinda perioperatif mortalite 10000 anestezide 3,0-15,9 arasinda değişmektedir ${ }^{30,34-36}$. Hem gelişmiş ülkelerde hem de gelişmekte olan ülkelerde perioperatif mortalite yenidoğan ve 1 yaş altı bebeklerde daha yüksek saptanmıştır ${ }^{37}$. ASA 3 ve üzerindeki hastalar ve acil operasyon ihtiyacı olan hastalar pediatrik populasyonda da önemli risk faktörleri olarak belirlenmiştir ${ }^{4,29,30,3,4,36}$ 88-40 . Anestezi tipleri incelendiğinde genel anestezi uygulanan hastalarda nöroaksiyal anesteziye göre daha sık kardiyak arrest ve mortalite gözlendiği saptanmıştıtr, ${ }^{6,1,30,39}$. Bazı çalışmalar pediatrik populasyonda solunum ve 
kardiyovaskler sistemin kardiyak arrest ve mortaliteye eşit oranda sebep olduğunu belirtirken bazı çalışmalarda solunumsal problemler, diğerlerinde kardiyovasküler problemler ön plana çıkmaktadır ${ }^{6,1,3,30}$. Kardiyovasküler etkilere en sık sebepler ise anestezik ilaç uygulamaları, kanama ve anemide yetersiz kan desteği olarak saptanmaktadır ${ }^{14}$.

\section{Yaşlı Populasyonda Perioperatif Kardiyak Arrest ve Ölümlerin İncelenmesi}

Yaşlı hastalarda kronik hastalıklar, kardiyovasküler hastalıklar ve fizyolojik yetersizlik tüm tıbbi tedavi ve müdahalelerde olduğu gibi cerrahi operasyonlarda da riski artırmaktadır $^{4 \prime}$. Yapılan çalışmalar yaşlı populasyonda genç erişkin populasyonla karşılaştırıldığından perioperatif kardiyak arrest ve mortalitenin daha yüksek olduğunu göstermiştir ${ }^{12,}$ 16, 32. Bubes ve arkadaşlarının yaptığı araştırmada 18367 adet 60 yaş üstü hasta incelenmiştir. 1996-2010 yılları incelendiğinde anesteziye bağ $\mathrm{l}_{\iota}$ kardiyak arrest insidansı 10000 anestezide 3,26 saptanırken mortalite 1,63 saptanmıştır. Kardiyak arrest nedenleri de anestezik ilaca bağlı veya hava yoluna bağlı problemler olarak saptanmıştır Yaşlı populasyonda en önemli intraoperatif kardiyak arrest belirteçleri ASA yüksekliği ve acil operasyonlar olarak saptanmıştır ${ }^{42} .2007$ yılında Tayland'da yapılan bir araştırma ise 65 yaş üstü hastalarda perioperatif mortaliteyi 10000 anestezide 39,3 olarak saptamıştır. Bu çalışma başlıca risk faktörlerini ASA skoru, acil operasyonlar ve kullanılan anestezik ilaçlar olarak belirlemiştir ${ }^{43}$. 70 yaş ve üzeri cerrahi hastalarının incelendiği bir çalışmada mortaliteye neden olan ameliyat öncesi faktörlerin en önemlileri yaş, ASA skorunun artışı, ameliyat öncesi albümin düzeyi düşüklüğü, acil operasyonlar olarak saptanmıştır ${ }^{4}$.

\section{Sonuç}

Perioperatif kardiyak arrest ve mortalite cerrahi operasyonlarm en önemli ve korkulan komplikasyonudur. Yapılan çalışmalar göstermiştir ki en riskli populasyon yenidoğan, 1 yaş altı çocuklar ve geriatrik populasyondur. En önemli risk faktörlerinde ise ASA skoru yüksekliği ve acil operasyonlar ön plana çımaktadır. Monitorizasyon tekniklerinin gelişmesi ve uygulamada kılavuzlara bağlı kalarak standardizasyon sağlanması muhakkak perioperatif mortalitenin azalmasın sağlayacaktır. Zaten yıllar içindeki perioperatif kardiyak arrest ve mortalite insidansındaki düşüş bunun göstergesidir. Anesteziye bağlı kardiyak arrestlerin major nedenlerinde ise hava yolu ve kullanılan ilaçlara bağlı komplikasyonlar öne çıkmaktadır. Bu konularm üstüne gidilmesi ve düzeltme stratejileri geliştirilmesi faydalı olacaktır.

\section{Kaynaklar}

1.Finks JF, Osborne NH, Birkmeyer JD. Trends in hospital volume and operative mortality for highrisk surgery. N Engl J Med 2011;364: 2128-37 2.Beecher HK, Todd DP. A study of the deaths associated with anesthesia and surgery:based on a study of 599,548 anesthesias in ten institutions 1948-1952, inclusive. Ann Surg 1954;140:32-5. 
3.Lagasse RS. Anesthesia safety: model or myth? A review of the published literature and analysis of current original data. Anesthesiology 2002;97:1609-17.

4.Newland MC, Ellis SJ, Lydiatt CA, et al. Anesthetic-related cardiac arrest and its mortality: a report covering 72,959 anesthetics over 10 years from a US teaching hospital. Anesthesiology 2002;97:108-15.

5.Holland R. Anesthetic mortality in New South Wales. Br J Anaesth 1987;59:834-41.

6.Keenan RL, Boyan CP. Cardiac arrest due to anesthesia. A study of incidence and causes. JAMA. 1985;253:2373-7.

7.Olsson GL, Hallén B. Cardiac arrest during anesthesia. A computer-aided study in 250,543 anesthetics. Acta Anaesthesiol Scand. 1988;32:65364 .

8.Aubas S, Biboulet P, Daures JP, et al. Incidence and etiology of cardiac arrest occurring during the peroperative period and in the recovery room. Apropos of 102,468 anesthesia cases. Ann Fr Anesth Reanim. 1991;10:436-42.

9.Cohen MM, Duncan PG, Pope WDB, et al. A survey of 112,000 anesthetics at one teaching hospital (1975-83). Can Anaesth Soc J 1986;33:2231.

10.Richard L, Keenan CPB. Decreasing frequency of anesthetic cardiac arrests. J Clin Anesth. 1991;3:354-7.

11.Sprung J, Warner ME, Contreras MG, et al. Predictors of survival following cardiac arrest in patients undergoing noncardiac surgery: a study of 518,294 patients at a tertiary referral center. Anesthesiology. 2003;99:259-69.

12.Goswami S, Brady JE, Jordan DA, et al. Intraoperative cardiac arrests in adults undergoing noncardiac surgery: incidence, risk factors, and survival outcome. Anesthesiology. 2002;117:101826.

13.Catré D, Lopes MF, Viana JS, et al. Perioperative morbidity and mortality in the first year of life: a systematic review (1997-2012). Rev Bras Anestesiol. 2015;65:384-94.

14.Morray JP, Geiduschek JM, Ramamoorthy C, et al. Anesthesia-related cardiac arrest in children: initial findings of the pediatric perioperative cardiac arrest (POCA). Reg Anesth. 2000;93:6-14.

15.Katz R, Lagasse R. Factors influencing the reporting of adverse outcomes to a quality management program. Anesth Analg. 2000;90:34450 .

16.Braz LG, Modolo NS, do Nascimento Jr P, et al. Perioperativecardiac arrest: a study of 53,718 anaesthetics over 9 yr from a Brazilian teaching hospital. Br J Anaesth. 2006;96:569-75.

17.Chu KM, Ford N, Trelles M. Operative mortality in resource-limited settings: the experience of Medecins Sans Frontieres in 13 countries. Arch Surg. 2010; 145: 721-25.
18.McIntyre T, Zenilman ME. Globalization of surgery: let's get serious. Arch Surg. 2010; 145: 715-6.

19.Gaba DM. Anesthesiology as a model for patient safety in health care. BMJ. 2000;320:785-8.

20.Cooper JB, Gaba D. No myth: anesthesia is a model for addressing patient safety. Anesthesiology. 2002;97:1335-7.

21.Lagasse RS: Apples and oranges: The fruits of labor in anesthesia care. Anesthesiology. 2003; 99:248-50.

22.Bishop MJ, Souders JE, Peterson CM, et al. Factors associated with unanticipated day of surgery deaths in Department of Veterans Affairs hospitals. Anesth Analg. 2008; 107:1924-35.

23.Braz LG, Braz DG, Cruz DS, et al. Mortality in anesthesia: a systematic review. Clinics. (Sao Paulo). 2009;64(10):999-1006.

24.Bainbridge D, Martin J, Arango M, et al. Evidence-based Peri-operative Clinical Outcomes Research (EPiCOR) Group. Perioperative and anaesthetic-related mortality in developed and developing countries: a systematic review and metaanalysis. Lancet. 2012;380(9847):1075-81.

25.Ellis SJ, Newland MC, Simonson JA, et al. Anesthesia-related cardiac arrest. Anesthesiology. 2014 Apr;120(4):829-38.

26.Sobreira-Fernandes D, Teixeira L, Lemos TS, et al. Perioperative cardiac arrests - A subanalysis of the anesthesia -related cardiacarrests and associated mortality. J Clin Anesth. 2018 Nov;50:78-90.

27.Morita K, Kawashima Y, Irita K, et al. Perioperative mortality and morbidity in 1999 with a special reference to age in 466 certified training hospitals of Japanese Society of Anesthesiologists report of Committee on Operating Room Safety of Japanese Society of Anesthesiologists. Masui. 2001;50(8):909-21.

28. Morita K, Kawashima Y, Irita K, et al. Perioperative mortality and morbidity in the year 2000 in 520 certified training hospitals of Japanese Society of Anesthesiologists: with a special reference to age-report of Japanese Society of Anesthesiologists Committee on Operating Room Safety. Masui. 2002;51(11):1285-96.

29.Flick RP, Sprung J, Harrison TE, et al. Perioperative cardiac arrests in children between 1988 and 2005 at a tertiary referral center: a study of 92,881 patients. Anesthesiology. 2007;106(2):226-37.

30.Bharti N, Batra YK, Kaur H. Paediatric perioperative cardiac arrest and its mortality: database of a 60-month period from a tertiary care paediatric centre. Eur J Anaesthesiol. 2009;26(6):490-5.

31.van der Griend BF, Lister NA, McKenzie IM, et al. Postoperative mortality in children after 101,885 anesthetics at a tertiary pediatric hospital. Anesth Analg. 2011;112(6):1440-7. 
32.Biboulet P, Aubas P, Dubourdieu J, et al. Fatal and non fatal cardiac arrests related to anesthesia. Can J Anaesth. 2001;48(4):326-32.

33. Murat I, Constant I, Maud'huy H. Perioperative anaesthetic morbidity in children: a database of 24,165 anaesthetics over a 30-month period. Paediatr Anaesth. 2004;14(2):158-66.

34.Bunchungmongkol N, Punjasawadwong Y, Chumpathong S, et al. Anesthesia-related cardiac arrest in children: the Thai Anesthesia Incidents Study (THAI Study). J Med Assoc Thai. 2009;92(4):523-30.

35.TayCL, TanGM,Ng SB.Critical incidents in paediatric anaesthesia: an audit of $10 \quad 000$ anaesthetics in Singapore. Paediatr Anaesth. 2001;11(6):711-8.

36.Ahmed A, Ali M, Khan M, et al. Perioperative cardiac arrests in children at a university teaching hospital of a developing country over 15 years. Paediatr Anaesth. 2009;19(6):581-6.

37.Gonzalez LP, Pignaton W, Kusano PS, et al. Anesthesia-related mortality in pediatric patients: a systematic review. Clinics (Sao Paulo). 2012;67(4):381-7.

38.Tiret L, Nivoche Y, Hatton F, et al. Complications related to anaesthesia in infants and children. A prospective survey of 40240 anaesthetics. Br J Anaesth. 1988;61(3):263-9.
39.Braz LG, Braz JR, Módolo NS, et al. Perioperative cardiac arrest and its mortality in children. A 9-year survey in a Brazilian tertiary teaching hospital. Paediatr Anaesth. 2006;16(8):860-6.

40.Bhananker SM, Ramamoorthy C, Geiduschek JM, et al. Anesthesia-related cardiac arrest in children: update from the Pediatric Perioperative Cardiac Arrest Registry. Anesth Analg. 2007;105(2):344-50.

41.Ward SA, Parikh S, Workman B.Health perspectives: international epidemiology of ageing. Best Pract Res Clin Anaesthesiol. 2011;25:305-17. 42.Nunes JC, Braz JR, Oliveira TS, et al. Intraoperative and anesthesia-related cardiac arrest and its mortality in olderpatients: a 15-year survey in a tertiary teaching hospital. PLoS One. 2014 Aug 12;9(8):e104041.

43.Rodanant O, Hintong T, Chua-in W, et al. The Thai anesthesia incidents study (THAI Study) of perioperative death in geriatric patients. J Med Assoc Thai. 2007;90:1375-81.

44.Story DA, Leslie K, Myles PS. Complications and mortality in older surgical patients in Australia and New Zealand (the REASON study): a multicentre, prospective, observational study. Anaesthesia. 2010;65(10):1022-30. 